\title{
Specific Imaging of Intracellular Lipid Droplets Using a Benzothiadiazole Derivative with Solvatochromic Properties
}

Hanna Appelqvist, Kati Stranius, Karl Börjesson, Peter Nilsson and Christine Dyrager

The self-archived version of this journal article is available at Linköping University Electronic Press:

http:/ / urn.kb.se/ resolve?urn=urn:nbn:se:liu:diva- 138482

N.B.: When citing this work, cite the original publication.

Appelqvist, H., Stranius, K., Borjesson, K., Nilsson, P., Dyrager, C., (2017), Specific Imaging of Intracellular Lipid Droplets Using a Benzothiadiazole Derivative with Solvatochromic Properties, Bioconjugate chemistry, 28(5), 1363-1370. https:/ / dx.doi.org/ 10.1021/ acs.bioconjchem.7b00048

Original publication available at:

https:/ / dx.doi.org/ 10.1021/ acs.bioconjchem.7b00048

Copyright: American Chemical Society

http:// pubs.acs.org/ 


\title{
Specific Imaging of Intracellular Lipid Droplets using a Benzothiadiazole Derivative with Solvatochromic Properties
}

\author{
Hanna Appelqvist, ${ }^{1}$ Kati Stranius, ${ }^{2}$ Karl Börjesson, ${ }^{2}$ K. Peter. R. Nilsson, ${ }^{1}$ \\ and Christine Dyrager*,1
}

${ }^{1}$ Department of Physics, Chemistry and Biology, Linköping University, 58183 Linköping, Sweden
${ }^{2}$ Department of Chemistry and Molecular Biology, University of Gothenburg, 41296 Göteborg,
Sweden

\begin{abstract}
Altered lipid metabolism and extensive lipid storage in cells has been associated with various medical disorders, including cancer. The development of fluorescent probes that specifically accumulates in lipid deposits are therefore of great interest in order to study pathological processes that are linked to dysregulated lipogenesis. In the present study, we present a small fluorescent benzothiadiazole dye that specifically stains lipid droplets in living and fixated cells. The photophysical characterization of the probe revealed strong solvatochromic behaviour, large Stokes shifts and high fluorescent quantum yields in hydrophobic solvents. In addition, the fluorophore exhibits a nontoxic profile and a high signal to noise ratio in cells (i.e. lipid droplets vs. cytosol), which make it an excellent candidate for studying lipid biology using confocal fluorescent microscopy.
\end{abstract}

\section{Introduction}

Intracellular lipid droplets (LDs) are lipid-rich spherical organelles that mainly consist of triglycerides (TAG) and cholesterol esters (CE) enclosed by a phospholipid monolayer with associated proteins. ${ }^{1-5}$ Besides being energy reservoirs, and providing building blocks for membrane synthesis, LDs are dynamic bodies with imperative cellular assignments ranging from membrane trafficking, fusion, and involvement in signal transduction pathways. ${ }^{6-10}$ Thus, the interest in LDs has emerged during recent years due to their association with pathological processes in diseases such as cancer, obesity, diabetes and inflammatory disorders. ${ }^{11-14}$ The development of fluorophores that specifically assembles in lipid deposits are therefore of great importance for studying dysregulated lipogenesis on a cellular level. Several lipid droplet specific dyes have been reported in the literature, such as Nile Red and various BODIPY derivatives. ${ }^{15-19}$ However, Nile Red also stains other hydrophobic structures in the cell, resulting in low LD selectivity and unfavourable signal-to-noise ratio. ${ }^{20-21}$ On the contrary, BODIPY fluorophores have shown high selectivity toward LDs. However, they usually display small Stokes shifts that can give background artifacts due to light scattering originating from the excitation source. ${ }^{22}$ Nevertheless, numerous efforts have been performed in order overcome these issues and this has further resulted in alternative LD-specific fluorophores with beneficial photophysical properties. ${ }^{23-27}$

In our search for fluorescent benzothiadiazole derivatives useful in studying cellular changes associated with malignant transformation, we came across an interesting compound that showed specific accumulation in intracellular lipid droplets. Hence, in this paper we investigate a fluorescent benzothiadiazole dye, hereinafter referred to as LD-BTD1 (Lipid Droplet-Benzothiadiazole 1), which could be used to explore pathological processes associated with extensive intracellular lipid storage. Notably, 
this compound has recently been reported in the literature, including synthesis, crystal structure determination and optical properties in dichloromethane. ${ }^{28}$ However, in the present study, we have characterized the photophysical properties for the derivative in solvents with different polarity and also investigated its specific localization in fibroblasts and melanoma cells using confocal fluorescence microscopy.

\section{Results and Discussion}

The benzothiadiazole (BTD) moiety has a strong electron-withdrawing capacity, and it is known for its favorable photophysical properties, such as large Stokes shifts, high signal-to-noise ratios and red-shifted optical profiles. ${ }^{29}$ The BTD unit has therefore extensively been used in polymeric materials for various light technology applications, primarily in organic light emitting diodes (OLEDs) and high performance solar cells. ${ }^{30}$ However, the use of BTDs in other research areas has rapidly been developed. For example, in the field of fluorescent cell microscopy, small synthetic modifications (e.g. introduction of different substituents) on the BTD scaffold have shown to generate fluorophores with unique properties in terms of photophysics, cell permeability and specific organelle accumulation. ${ }^{29}$ For instance dyes that exclusively assemble in mitochondria, the cytosol, the nucleus (due to e.g. binding to double-stranded DNA) or in lipid-based structures. ${ }^{31-35}$

Synthesis: In the present study, we have combined the electron-withdrawing benzothiadiazole scaffold with an electron-donating substituent (dimethylaminophenyl), resulting in a push-pull fluorophore. ${ }^{28}$ In a push-pull system, the LUMO is mainly localized on the acceptor moiety whereas the HOMO is centered on the donor fragment. This allows for individual tuning of the HOMO and LUMO energies, resulting in a low HOMO-LUMO gap, and thus a red-shifted absorption. ${ }^{36}$ As depicted in Scheme 1, the benzothiadiazole derivative (LD-BTD1) was prepared in $96 \%$ yield using a palladium mediated Suzuki reaction. Accordingly, 4-bromo2,1,3-benzothiadiazole and 4-(dimethylamino)phenylboronic acid were cross-coupled in the presence of PEPSSI-IPr as catalyst and $\mathrm{K}_{2} \mathrm{CO}_{3}$ as base in Toluene/MeOH (1:1).

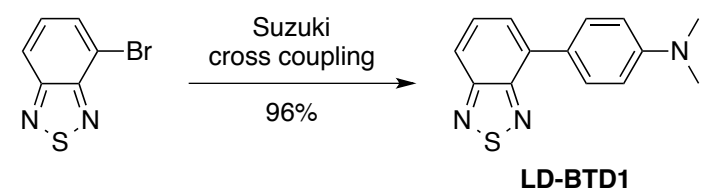

Scheme 1. Reagents and conditions: 4-(dimethylamino)phenylboronic acid, PEPPSI-Ipr, $\mathrm{K}_{2} \mathrm{CO}_{3}$, Toluene/MeOH $(1: 1), 80{ }^{\circ} \mathrm{C}$, overnight.

Cellular Studies: Initially, we wanted to explore the utility of LD-BTD1 as a fluorophore for cell imaging using confocal fluorescence microscopy. Human normal fibroblasts from the skin (AG01518) and a malignant transformed cell line originating from melanocytes in the skin (malignant melanoma, SK-MEL-28) were used in the study. LD-BTD1 staining of cultured cells, both living and fixated, resulted in a punctate staining pattern (Figure 1A and Supplementary Figure S1). The staining was more intense in the melanoma cell line compared to normal fibroblasts, although staining was also seen in the fibroblasts when using a higher gain (Supplementary Figure S2). The evident difference in fluorescence intensity was quantified by flow 
cytometry, showing that the median fluorescence intensity in melanoma cells was significant higher compared to the intensity in the fibroblasts (Figure 1B-D), which is well in accordance with the microscopy data.

A

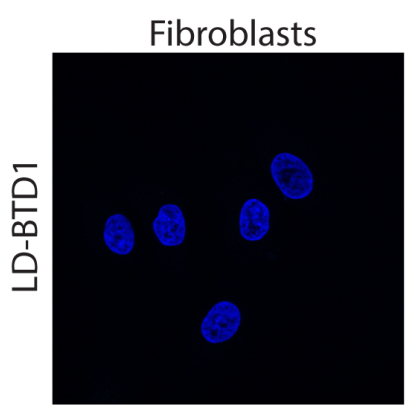

Melanoma cells

B

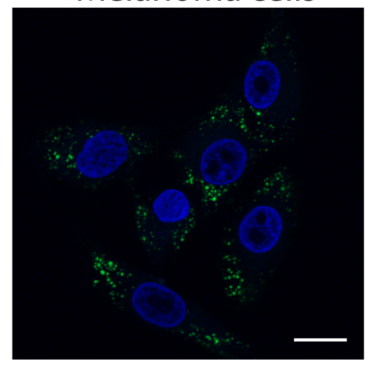

C
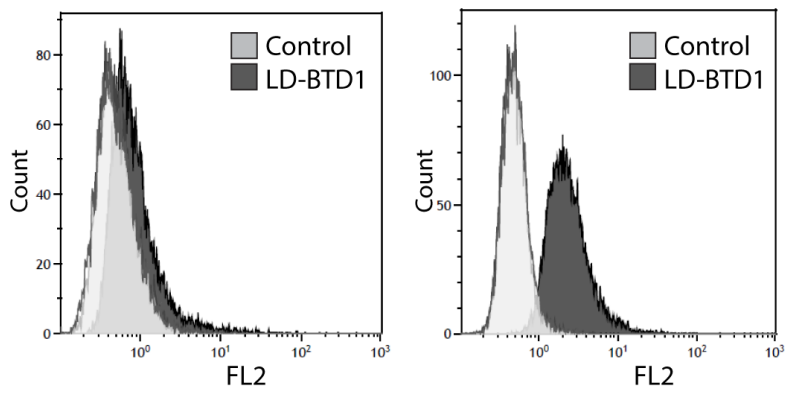

D

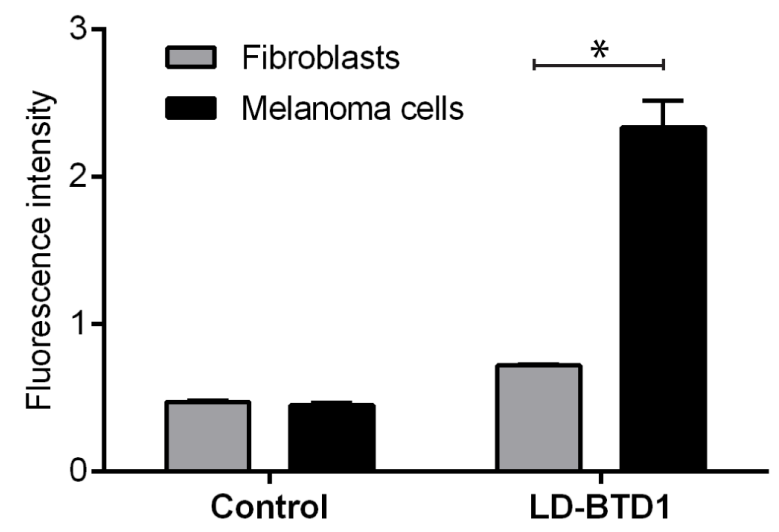

Figure 1. Human fibroblasts and melanoma cells incubated with LD-BTD1 $(10 \mu \mathrm{M})$ for $24 \mathrm{~h}$. A) Confocal microscopy images of cells stained with LD-BTD1 (seen in green; ex $458 \mathrm{~nm}$, em 465-682 $\mathrm{nm}$ ) and DAPI (nuclei staining seen in blue; ex 405, em 410-453). Scale bar $10 \mu \mathrm{m}$. Staining was performed on living cells, which were fixated directly after the incubation (prior to the microscopy analysis). Panels B and C show representative histograms from flow cytometry analysis of LD-BTD1 in fibroblasts and melanoma cells, respectively. D) Quantification (median fluorescence intensity) of the data presented in $\mathrm{B}$ and $\mathrm{C}$. Results are presented as mean $+\mathrm{SD} ; \mathrm{n}=4 . *$ denotes statistical significant difference $(\mathrm{p} \leq 0.05)$.

To further explore the potential of LD-BTD1 as a fluorophore for cell imaging, the cell viability during prolonged incubation $(72 \mathrm{~h})$ was investigated using the MTTassay. ${ }^{37}$ No signs of toxicity could be seen in the cell cultures after incubation with LD-BTD1, as cell morphology was indifferent from controls (Figure 2A). In addition, no significant alteration in cell viability was observed (Figure 2B), suggesting that LD-BTD1 is non-toxic at $10 \mu \mathrm{M}$ and possible to use in living cells for prolonged 
times. However, staining was also analyzed at lower concentrations, which revealed a detection limit of approximately $0.5 \mu \mathrm{M}$ (Supplementary figure S3).

A

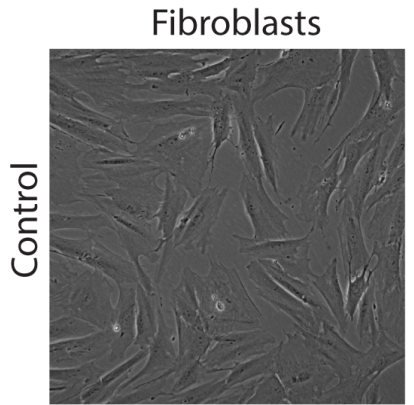

Melanoma cells
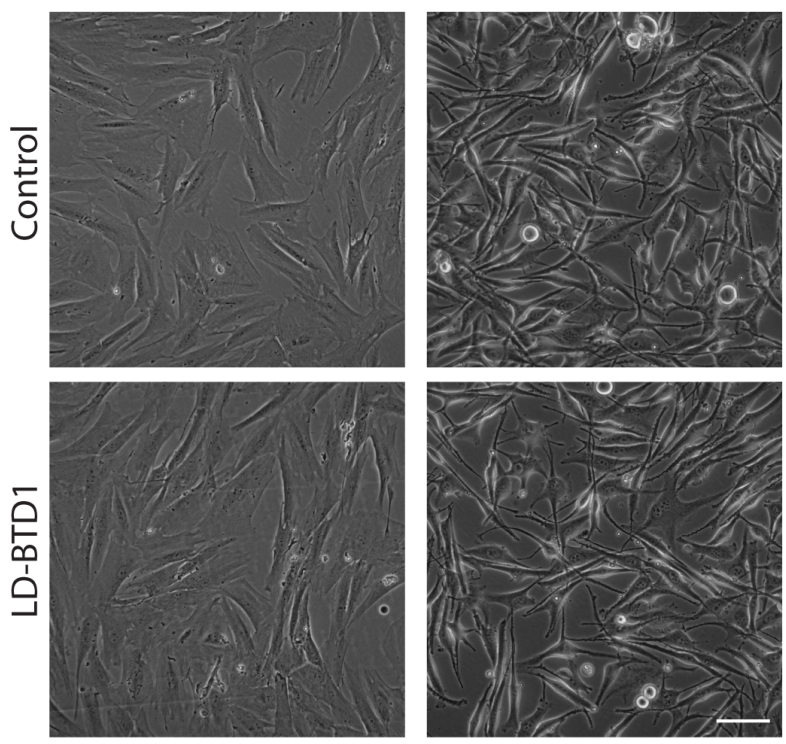

B

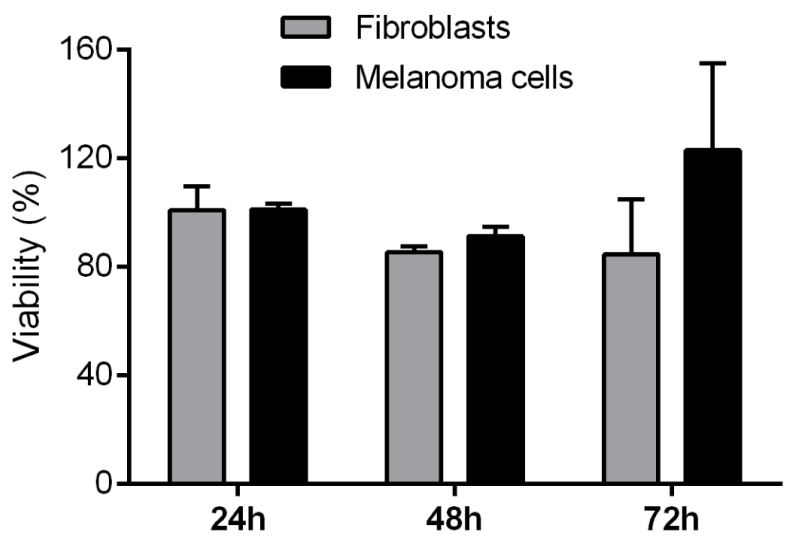

Figure 2. Cell viability data of human fibroblasts and melanoma cells incubated with LD-BTD1 (10 $\mu \mathrm{M})$. A) Phase-contrast images of cells treated with LD-BTD1 for $48 \mathrm{~h}$ (scale bar $40 \mu \mathrm{m}$ ). B) Cell viability, measured by the MTT assay, after treatment with LD-BTD1 for up to $72 \mathrm{~h}$. Results are expressed as \% of corresponding DMSO control and presented as mean+ SD; $\mathrm{n}=4$.

The punctate intracellular pattern for LD-BTD1 was further investigated using costaining with different markers for major cellular organelles. The results did not show any apparent co-localization with mitochondria, lysosomes, early endosomes or autophagosomes (Supplementary Figure S4). However, specific staining of hydrophobic compartments (including LDs) using Nile Red showed an equivalent staining pattern as for LD-BTD1 (Supplementary Figure S5). Notably, co-localization analysis of LD-BTD1 and Nile Red is not possible since their excitation and emission spectra overlap (Supplementary Figure S6) and thereby cannot be distinguished by fluorescence microscopy. Instead, LD-BTD1 staining was combined with an antibody towards adipophilin. Adipophilin, also known as perilipin2 (PLIN2) or adipose differentiation-related protein (ADRP), is a protein that associate with the lipid droplet surface and is therefore widely utilized as a protein marker for LDs. ${ }^{38}$ As 
shown in Figure 3, we found that LD-BTD1 and adipophilin co-localized in the cells. Thus, in panels to the right (Figure 3, merged and zoomed) adipophilin staining is seen in the periphery of LD-BTD1-positive dots, which is well in concordance with adipophilin being localized on the surface of the LDs. These results indicate that LDBTD1 accumulates in LDs in cultured cells and further suggests that it preferentially stains the core of the LD, where the hydrophobic triglycerides and cholesterol esters are found.

In general, LDs can virtually be found in any cell. However, many cell types exhibit only a few, small lipid droplets under normal conditions, while others (such as adipocytes or hepatocytes) have a higher content due to active lipid synthesis and storage. $^{39}$ A low level of LDs is a reasonable explanation for the relative weak staining of LD-BTD1 seen in fibroblasts (Figure 1A). Further, upregulated lipogenesis is a common characteristic in a number of human carcinomas, which might explain the higher staining intensity observed in the malignant melanoma cell line. ${ }^{40-41}$ In order to endorse the LD specific staining of LD-BTD1, we performed experiments on human isolated adipocytes (Supplementary figure S7), which often contain one single large LD. ${ }^{1}$ LD-BTD1 staining of adipocytes revealed staining that corresponded to a large LD constituting the vast majority of the cell area and displacing the nucleus to the periphery (Supplementary Figure S7), which is well in concordance with previous reports. ${ }^{42-43}$
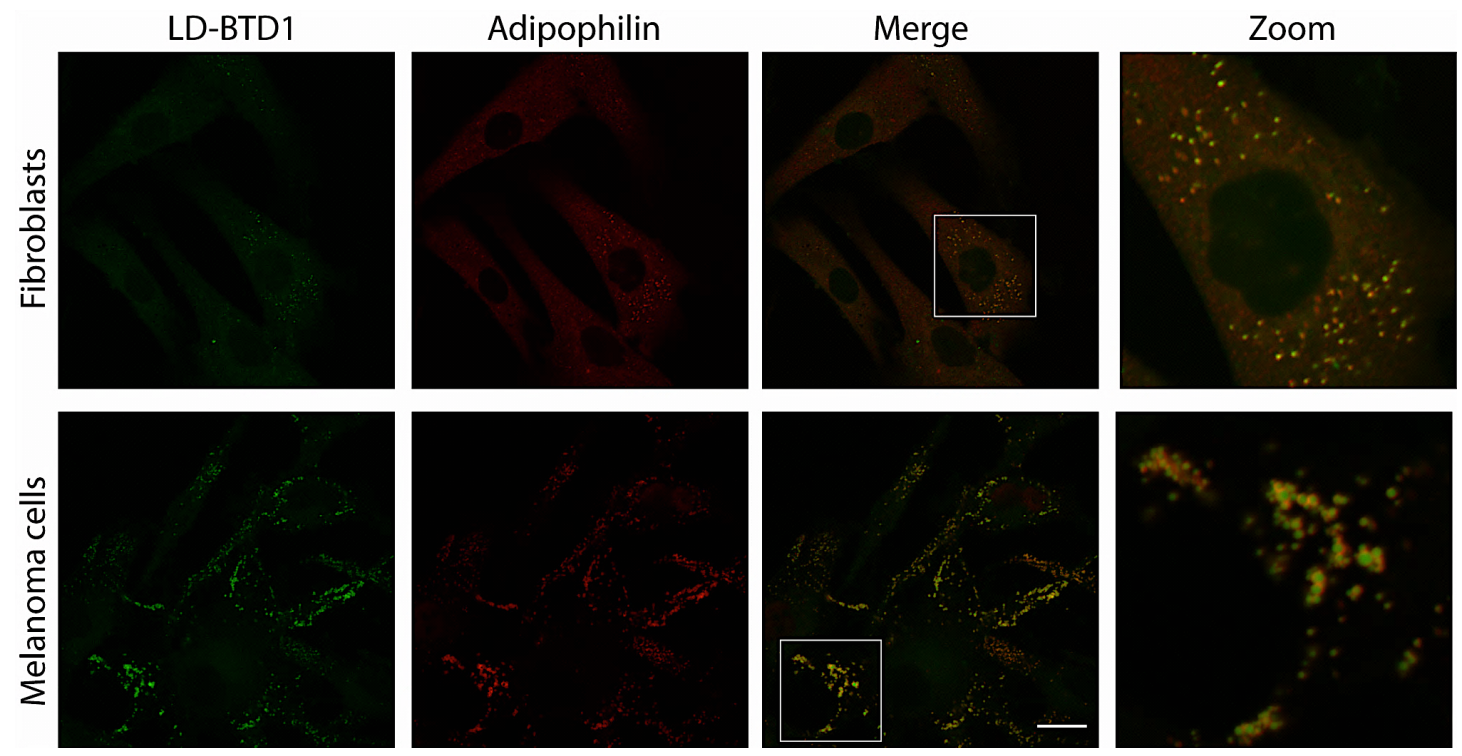

Figure 3. Fluorescent microscopy images of LD-BTD1 $(10 \mu \mathrm{M}, 24 \mathrm{~h})$ and the lipid droplet associated protein adipophilin (PLIN-2) in fibroblasts and melanoma cells. Staining was performed on living cells, which were fixated directly after the incubation and further used for immunocytochemistry. Panels to the left show LD-BTD1 (seen in green; ex $458 \mathrm{~nm}$, em 484-707 nm) or the anti-PLIN-2 antibody (seen in red; ex $595 \mathrm{~nm}$, em 600-734 nm). The panels to the right show merged images in order to emphasize the co-localization between LD-BTD1 and the adipophilin antibody. Scale bar 10 $\mu \mathrm{m}$.

Supplementation of the cell culture media with fatty acids (e.g. oleic acid) is known to enhance the number and size of LDs, due to the stimulation of the fatty acid receptor FFAR4. ${ }^{38,44}$ These dynamic features of LDs can be visualized using a fluorophore that specifically accumulates in lipid deposits. As shown in Figure 4, the increased quantity of LDs upon the addition of oleic acid could clearly be monitored by 
enhanced fluorescence intensity, both in fibroblasts and melanoma cells, using contemporary staining with LD-BTD1. Notably, the dynamics of LDs has recently been studied in living cells using a benzothiadiazole derivative with a pendant oleic acid tail. ${ }^{35}$ However, our results demonstrate that the small LD-BTD1 derivative (in the absence of a fatty acid substituent) is a suitable fluorophore for studying dynamic changes in LD biology. Altered lipid metabolism and extensive lipid storage, represented by enhanced numbers of LDs, have been described in several neoplastic diseases and are considered as a hallmark of cancer aggressiveness and poor diagnosis. $^{12,41,45}$ Besides the accumulation in cancer cells, LD alterations are frequently observed in infections and other inflammatory disorders. ${ }^{12}$ Therefore, LDBTD1 is a potential LD dye that could be used in preclinical studies of cancer as well as in the study of inflammatory diseases.

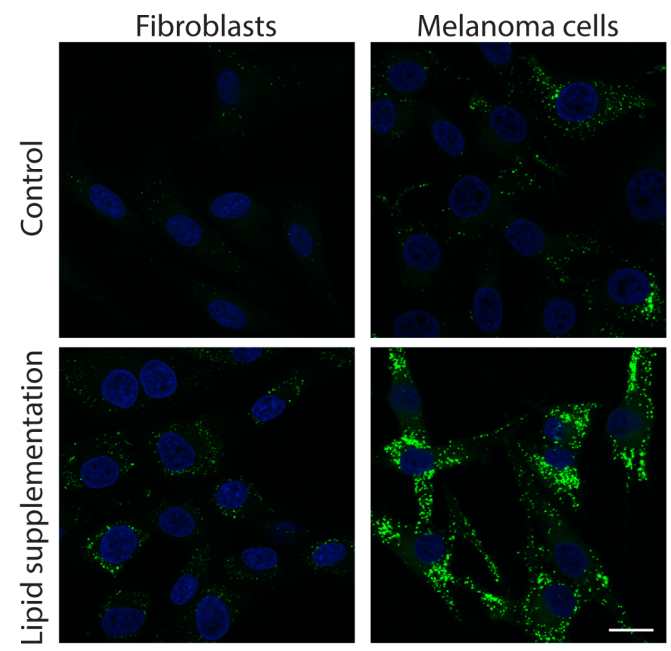

Figure 4. LD-BTD1 staining (seen in green; ex $458 \mathrm{~nm}$, em 465-682 nm) in fibroblasts and melanoma cells after lipid supplementation with oleic acid $(150 \mu \mathrm{M}, 24 \mathrm{~h})$. Cell nuclei are stained with DAPI (seen in blue; ex $405 \mathrm{~nm}$, em 410-453 nm). Scale bar $10 \mu \mathrm{m}$. Staining was performed on living cells, which were fixated directly after the incubation (prior to the microscopy analysis).

Photophysical Characterization: In order to rationalize the selective emission of LD-BTD1 in cells, the photophysical properties of LD-BTD1 were explored by UVvis absorption and emission spectroscopy. In hexane, LD-BTD1 exhibits an absorption maximum at $420 \mathrm{~nm}\left(\varepsilon=7100 \mathrm{M}^{-1} \mathrm{~cm}^{-1}\right)$, a fluorescence maximum at 511 $\mathrm{nm}$ (Figure 5) and a high fluorescence quantum yield $\left(\varphi_{\mathrm{F}}=0.66\right)$. The brightness $(\alpha$ extinction coefficient $*$ fluorescence quantum yield) is proportional to the fluorescence output per chromophore and can be used as a value of merit between fluorescent probes. Consequently, the brightness of LD-BTD1 in hexane could be described as moderate due to its relatively low extinction coefficient. However, the highest priority for cellular microscopy applications is to acquire a large signal-tonoise ratio between the studied species and the background. Factors that can affect the signal-to-noise ratio include: 1) the concentration ratio of the probe between the cellular feature under study and the surroundings, 2) the difference in the fluorescence quantum yield of the probe between the cellular target under study and the surroundings, and 3) the magnitude of the Stokes shift of the fluorophore, as to avoid scattered light from the excitation source. 


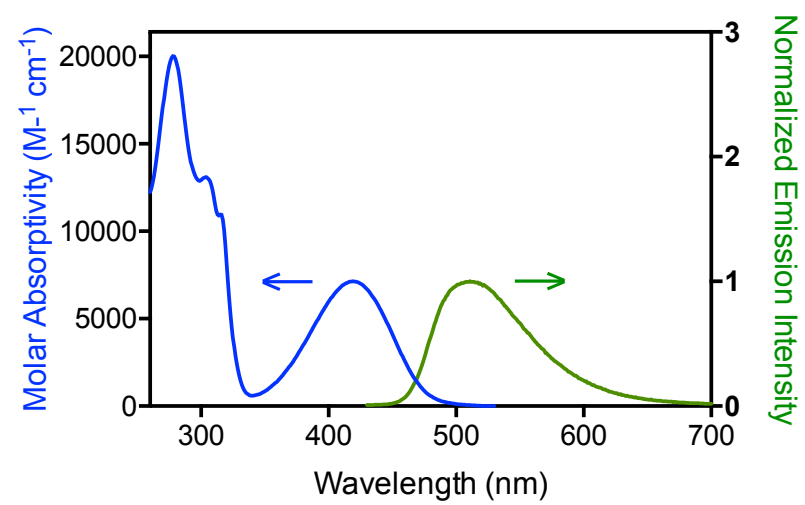

Figure 5. Molar absorptivity (blue) and normalized emission spectra (green, exc.430 nm) of LD-BTD1 in hexane.

To give a rough estimate of the partitioning equilibrium between LD-BTD1 in water and lipid droplets, the octanol-water partition constant, $\mathrm{K}_{\mathrm{ow}}$, was measured. The $\mathrm{K}_{\mathrm{ow}}$ was determined to 2000 , which corresponds to $\log \mathrm{P}=3.3$. However, due to the relative difficulty in measuring high $\mathrm{K}_{\mathrm{ow}}$ values, this should be viewed as a lower estimate. The $\mathrm{K}_{\mathrm{ow}}$ value can be used for direct estimation of the concentration ratio of LD-BTD1 between a lipid feature and the cytosol. Thus, when using LD-BTD1 as a fluorescent probe in a cellular experiment, the concentration ratio between the lipid feature and the cytosol is expected to be greater than 2000. To give an idea on the difference on the photophysical properties of LD-BTD1 in a hydrophobic and lipidrich environment compared to the cytosol, the general solvatochromic behavior of LD-BTD1 was investigated in a series of solvents with various polarity index. Absorption and fluorescence maxima (Supplementary Figure S8 and S9), Stokes shift values and fluorescence quantum yields for LD-BTD1 in solvents of different polarity are presented in Table 1. LD-BTD1 exhibits large solvatochromic shifts with increasing solvent polarity from hexane $\left(\lambda_{\mathrm{E}, \max }=511 \mathrm{~nm}\right)$ to $\operatorname{DMSO}\left(\lambda_{\mathrm{E}, \max }=759 \mathrm{~nm}\right)$. A Lippert plot (Supplementary Figure S10) was used to rationalize the solvent effect on spectral shifts for LD-BTD1. The linearity of this plot indicates that the solvent polarity is the dominant reason for spectral shifts, and thus, that the dipole moment of the ground and excited state differs significantly. ${ }^{46}$ Specific solvent effects were also investigated by the addition of a small amount of water to LD-BTD1 dissolved in DMF (Supplementary Figure S11). Increasing amount of water (0.5-10\%) did not result in significant spectral changes, except for emission quenching. This excludes specific solvent effects, such as hydrogen bonding, as a reason for the observed spectral shifts. Furthermore, the spectroscopic properties in water were also investigated. However, $10 \%$ DMSO in water was used for the measurements due to the low solubility in water. Despite of increasing the polarity with a quite large percentage, considerable blue-shifts were seen in the absorption and emission spectra (Supplementary Figure 12), suggesting that the dye is aggregating in an aqueous environment. 
Table 1. Absorption and fluorescence maxima, Stokes shift values and fluorescence quantum yields for LD-BTD1 in solvents of different polarity.

\begin{tabular}{lllll}
\hline Solvent & $\lambda_{\mathrm{A}, \max }(\mathrm{nm})$ & $\lambda_{\mathrm{E}, \max }(\mathrm{nm})$ & $\lambda_{\mathrm{E}, \max }-\lambda_{\mathrm{A}, \max }(\mathrm{nm})$ & $\varphi_{\mathrm{F}}$ \\
\hline Hexane & 420 & 511 & 91 & 0.66 \\
Toluene & 429 & 578 & 149 & 0.71 \\
Diethyl ether & 420 & 583 & 163 & 0.66 \\
THF & 429 & 633 & 204 & 0.38 \\
Octanol & 431 & 635 & 204 & 0.02 \\
Methanol & 423 & 709 & 286 & $<0.01$ \\
DMF & 429 & 713 & 284 & 0.04 \\
DMSO & 440 & 759 & 319 & 0.03 \\
\hline
\end{tabular}

The fluorescent quantum yields of LD-BTD1 were found to decrease from $70 \%$ to less than $1 \%$ going from low to high polarity solvents (Table 1). However, comparing solvents of similar polarity but with different capability for hydrogen bonding, such as THF and octanol, indicates that hydrogen bonding increases quenching of the excited state by contributing to the non-radiative relaxation process. This has also been earlier been observed in other benzothiadiazole containing fluorophores. $^{47-49}$ The two orders of magnitude difference of the fluorescence quantum yield between a hydrophobic and hydrophilic environment results in a high contrast ratio between LD-BTD1 being present in different cellular environments. Furthermore, the contrast ratio between the surroundings depends on the concentration ratio between the environments times the difference in fluorescence quantum yield. The observed contrast ratio at equilibrium between LD-BTD1 being present in the cytosol compared to a hydrophobic environment is thus expected to exceed $2 \times 10^{5}$ (i.e. the concentration multiplied with the difference in fluorescence quantum yield). Also, for microscopy studies, the relative high Stokes shift (even in hydrophobic solvents such as hexane) is advantageous, since it makes removal of scattered light from the excitation source straightforward. Furthermore, the $\mathrm{pH}$ dependence ( $\mathrm{pH}$ 2-12) of LD-BTD1 was investigated, which showed that the emission was not significantly changed in a wide range $(\mathrm{pH} \mathrm{4-12)} \mathrm{(Supplementary}$ Figure S13). However, a reduced intensity was observed at $\mathrm{pH} 2$, likely due to protonation of the benzothiadiazole. ${ }^{50-51}$ Importantly, the shape of the emission curve was unchanged at $\mathrm{pH} \mathrm{4-12}$ and we therefore suggest that the compound is stable under these conditions.

\section{Conclusion}

In summary, we have described the synthesis, photophysical characterization and confocal fluorescence microscopy studies of a small nontoxic benzothiadiazole derivative (LD-BTD1) that specifically stains intracellular lipid droplets. The fluorophore exhibits strong solvatochromic behavior in solvents with increasing polarity, resulting in Stokes shifts ranging from $91 \mathrm{~nm}$ (in hexane) to $319 \mathrm{~nm}$ (in DMSO). The localization of LD-BTD1 in the lipophilic environment of LDs can be rationalized by its preferred partition in hydrophobic solvents in comparison with an aqueous solution $\left(\mathrm{K}_{\mathrm{ow}}=2000\right)$. In addition, the fluorescence quenching in water and the high quantum yields in hydrophobic solvents (e.g. toluene $\varphi_{\mathrm{F}}=0.71$ ) further enlarges the perceived contrast between the LDs and the cytosol resulting in low to none background. We have further demonstrated that LD-BTD1 clearly can reflect the higher abundance of LDs in melanoma cancer cells in comparison to normal fibroblasts. It can also be used to study lipid dynamics in cells, which were shown using fatty acid supplementation. Thus, we foresee that LD-BTD1 can be used as an 
attractive fluorescent dye for exploring the role of extensive lipid storage and dynamics that are related to pathological processes in cancer and inflammatory disorders.

\section{Experimental Procedures}

\section{General}

All reagents and solvents were of analysis or synthesis grade and were used without further purification unless otherwise indicated. ${ }^{1} \mathrm{H}$ and ${ }^{13} \mathrm{C}$ NMR were recorded on a on a Varian 300 instrument (Varian Inc., Santa Clara, CA, USA) (at 300 and $75 \mathrm{MHz}$, respectively) in $\mathrm{CDCl}_{3}$. Chemical shift is reported in ppm with the solvent residual peak as reference; $\mathrm{CDCl}_{3}\left(\delta_{\mathrm{H}} 7.26, \delta_{\mathrm{C}} 77.0\right)$. The reaction was monitored by thin-layer chromatography (TLC), on silica plated (Silica gel $60 \mathrm{~F}_{254}$, E. Merck) aluminium sheets, detecting spots by UV (254 and $365 \mathrm{~nm})$. Flash chromatography was performed using Merck Silica Gel $60(40-63 \mu \mathrm{m})$. High-resolution mass data (ESToF) were obtained from the Division of Mass spectrometry, Department of Chemistry, Imperial College London. Absorbance was measured using a Cary 5000 (Varian) or Lambda 650 (Perkin Elmer) spectrophotometer. Fluorescence measurements were done using a Spex Fluorolog (JY Horiba) spectrofluorometer. Hexane, toluene, diethyl ether, THF and DMF were dried using an MBraun MB SPS800 solvent purification system. All other chemicals were used without further purifications.

\section{Synthesis of 4-(4-dimethylaminophenyl)-2,1,3-benzothiadiazole (LD-BTD1).}

PEPSSI-IPr $(8.0 \mathrm{mg}, 0.011 \mathrm{mmol})$ was added to a solution of 4-bromo-2,1,3benzothiadiazole $(0.123 \mathrm{~g}, 0.57 \mathrm{mmol})$, 4-(dimethylamino)phenylboronic acid $(0.189$ $\mathrm{g}, 1.15 \mathrm{mmol})$ and $\mathrm{K}_{2} \mathrm{CO}_{3}(0.237 \mathrm{~g}, 1.72 \mathrm{mmol})$ in Toluene/MeOH 1:1 (16 mL). The reaction was heated at $80{ }^{\circ} \mathrm{C}$ for $2 \mathrm{~h}$, was then allowed to reach room temperature and was further stirred overnight. The reaction mixture was added dropwise to water (50 $\mathrm{mL})$ and the solution was extracted with Ethyl acetate $(1 \times 30 \mathrm{~mL})$. The organic layer was washed with water $(2 \times 30 \mathrm{~mL})$, concentrated under reduced pressure and the residue was further recrystallized from $\mathrm{MeOH} /$ water to obtain a red solid. Purification using column chromatography (100\% Toluene to $10 \%$ Ethyl acetate in Toluene) gave the pure product as an orange power $(0.141 \mathrm{~g}, 96 \%) .{ }^{1} \mathrm{H}$ NMR $\left(300 \mathrm{MHz}, \mathrm{CDCl}_{3}\right) \delta$ $3.04(\mathrm{~s}, 6 \mathrm{H}), 6.85-6.91(\mathrm{~m}, 2 \mathrm{H}), 7.60-7.67(\mathrm{~m}, 2 \mathrm{H}), 7.86-7.92(\mathrm{~m}, 3 \mathrm{H}) ;{ }^{13} \mathrm{C}$ NMR $(75$ $\left.\mathrm{MHz}, \mathrm{CDCl}_{3}\right) \delta 40.4,112.3,118.9,125.2,125.8,129.8,130.0,134.7,150.6,153.7$, 155.8. HRMS (ES-ToF) $[\mathrm{M}+\mathrm{H}]^{+}$Calcd for $\mathrm{C}_{14} \mathrm{H}_{14} \mathrm{~N}_{3} \mathrm{~S}$, 256.0903. Found, 256.0915.

\section{Cell lines and culture conditions}

Normal human skin fibroblasts (AG01518; passages 12-24; Coriell Institute, Camden, NJ, USA) and malignant melanoma cells SK-MEL-28 (HTB-72; ATCC, Manassas, VA, USA) were cultured in Eagle's minimum essential medium (EMEM) GlutaMAX, supplemented with $50 \mathrm{IU} / \mathrm{ml}$ penicillin-G, $50 \mu \mathrm{g} / \mathrm{ml}$ streptomycin, and 10 $\%$ fetal bovine serum (all from Gibco, Paisley, UK). Cells were kept in humidified air with $5 \% \mathrm{CO}_{2}$ at $37{ }^{\circ} \mathrm{C}$. The day before experiments, cells were seeded to an approximate $50 \%$ confluence. For microscopic analysis cells were seeded on glass coverslips No 1.0. 


\section{Staining of cells}

The day after seeding, cells were stained with $10 \mu \mathrm{M}$ LD-BTD1 in complete cell culture media for indicated times. Notably, the staining solution $(10 \mu \mathrm{M})$ was prepared from a $1.5 \mathrm{mM}$ stock solution of LD-BTD1 in DMSO immediately prior to use, which correlates to a final DMSO concentration of $<1 \%$. In all experiments a corresponding DMSO control was run in parallel. For staining of fixed cells, cells were first fixed in $4 \%$ paraformaldehyde (PFA; $20 \mathrm{~min}, 4{ }^{\circ} \mathrm{C}$ ), rinsed three times with PBS and thereafter incubated with LD-BTD1 (500 nM, $30 \mathrm{~min}$; room temperature). For microscopic evaluation, the cells were rinsed three times with PBS, fixed in $4 \%$ paraformaldehyde (PFA; $20 \mathrm{~min}, 4{ }^{\circ} \mathrm{C}$ ) and mounted using Vectashield with or without DAPI (Vector Laboratories, Burlingame, CA, USA) for visualization of the cell nuclei.

\section{Isolation of primary human adipocytes}

The study was approved by the Regional Ethics Board at Linköping University, Sweden (00-398) and has been carried out in accordance with the Declaration of Helsinki. During elective surgery at the University Hospital in Linköping, Sweden, a piece of subcutaneous abdominal fat tissue was excised and immediately treated by collagenase (type 1, Worthington, NJ, USA) digestion in modified Krebs-Ringer solution $\left(0.12 \mathrm{M} \mathrm{NaCl}, 4.7 \mathrm{mM} \mathrm{KCl}, 2.5 \mathrm{mM} \mathrm{CaCl}_{2}, 1.2 \mathrm{mM} \mathrm{MgSO}_{4}, 1.2 \mathrm{mM}\right.$ $\left.\mathrm{KH}_{2} \mathrm{PO}_{4}\right)$ to isolate adipocytes. ${ }^{52}$ The cells were incubated overnight in the same solution mixed with DMEM containing $7 \%$ albumin, $200 \mathrm{nM}$ phenylisopropyladenosine, $20 \mathrm{mM}$ HEPES, $50 \mathrm{UI} / \mathrm{ml}$ penicillin, $50 \mu \mathrm{g} / \mathrm{ml}$ streptomycin, $\mathrm{pH} 7.40$, at $37^{\circ} \mathrm{C}$. LD-BTD1 was added to a final concentration of 10 $\mu \mathrm{M}$. The day after, the adipocytes were washed in PBS, fixed in PFA, added to polyL-lycine coated coverslips and mounted with DAPI.

\section{Viability Analysis}

The reducing capacity of cell cultures was measured using the 3-(4,5dimethylthiazol-2-yl)-2,5-diphenyltetrazolium bromide (MTT) reduction assay (Sigma-Aldrich). ${ }^{37}$ This method is widely used to assess cytotoxicity and cell viability, and it is currently thought that the amount of MTT formazan is proportional to the number of living cells. Cells were incubated with $0.5 \mathrm{mg} / \mathrm{ml}$ MTT for $2 \mathrm{~h}$ at $37^{\circ} \mathrm{C}$. Then, the MTT solution was removed and the formazan product was dissolved in DMSO. The absorbance was measured at $550 \mathrm{~nm}$ with a VICTOR ${ }^{\mathrm{TM}}$ X Series Multiple Plate Reader (PerkinElmer, Waltham, MA).

\section{Flow cytometry}

For flow cytometry, the stained cells were detached by trypsizination, washed with PBS, filtrated and analysed using a Gallios flow cytometer (Beckmancoulter,Gallios ${ }^{\mathrm{TM}}$, USA). The fluorescence was measured by using a 488 $\mathrm{nm}$ laser, using 595SP and 575BP filters (FL2). Ten thousand cells were collected for each sample and the data was analyzed using the software Kaluza.

\section{Co-staining with organelle markers}

For staining of mitochondria, probe-stained cells were incubated with MitoTracker Orange CMTMRos (150 nM, $30 \mathrm{~min}, 37^{\circ} \mathrm{C}$; Molecular Probes, Eugene, OR). Cells were then fixed in $4 \%$ PFA $\left(20 \mathrm{~min}, 4^{\circ} \mathrm{C}\right)$. For immunostaining, stained cells were after fixation permeabilized with $0.1 \%$ saponin (Sigma-Aldrich) in PBS containing 5 $\%$ fetal bovine serum (20 min, room temperature) and incubated for $2 \mathrm{~h}$ at room 
temperature with the monoclonal mouse primary antibodies: lysosome-associated membrane protein 2 (LAMP-2, 1:50; Southern Biotech, Birmingham, AL), early endosomal antigen-1 (EEA-1, 1:400; Sigma-Aldrich), LC3B (1:100, Novus Biologicals, Littleton, CO, USA) or adipophilin (ADRP; 1:50; Abcam, Cambridge, UK). This step was followed by incubation with the appropriate secondary antibodies conjugated to Alexa Fluor 594 (1:400, Molecular Probes) for $1 \mathrm{~h}$. For staining of lipid droplets, fixed cells were stained with $0.1 \mu \mathrm{g} / \mathrm{ml} \mathrm{Nile} \mathrm{Red} \mathrm{in} 150 \mathrm{mM} \mathrm{NaCl}, 10 \mathrm{~min}$ at room temperature. Cells were mounted using Vectashield. For microscopic analysis of the samples, an inverted LSM 780 confocal microscope (Carl Zeiss, Oberkochen, Germany) was used. Cells stained with LD-BTD1 and DAPI were analyzed using lasers at 458 and $405 \mathrm{~nm}$, respectively, and the emission settings were $465-682 \mathrm{~nm}$ and 410-453 $\mathrm{nm}$. The emission settings for LD-BTD1 in the co-localization experiments were 484-707 nm, except for the co-localization with mitochondria were 466-545 nm were used. Nile Red fluorescence was collected with the optimal excitation and emission settings, excitation $458 \mathrm{~nm}$ and emission 484-707 nm, for imaging of LDs over hydrophobic membranes (seen when using a more red-shifted wavelength). ${ }^{38}$ Cell cultures stained with Alexa594 conjugate antibodies were analyzed using a $595 \mathrm{~nm}$ laser for excitation and the emission was collected at 600$734 \mathrm{~nm}$ ). Mitotracker Orange CMTMRos was excited at $550 \mathrm{~nm}$ and the emission settings were 555-750 nm. A plan-Apochromat 633/1.40. Oil DIC 60 X M27 objective was used for all imaging.

\section{Lipid supplementation}

Accumulation of lipid droplets were stimulated by adding $150 \mu \mathrm{M}$ oleic acid-albumin (Sigma-Aldrich, St. Louis, MO, USA) to the cell culture medium and the cells were then incubated $24 \mathrm{~h}$ together with LD-BTD1.

\section{Statistical analysis}

All experiments were repeated four times and the results are presented as the means and standard deviations of independent samples. Data were statistically evaluated using student's t-test (comparison of two groups) or ANOVA. P values $\leq 0.05$ were considered to be significant. All calculations were done using SPSS Statistics 24.

\section{Quantum yield determination}

Fluorescent quantum yields $\left(\varphi_{\mathrm{F}}\right)$ of LD-BTD1 in different polarity solvents were measured relative to fluorescein in $20 \mathrm{mM} \mathrm{NaOH}(\mathrm{aq})\left(\varphi_{\mathrm{F}}=0.93\right) .{ }^{53}$ The excitation wavelength for the quantum yield measurements was chosen so that the absorbance was around 0.02-0.04 and so it was possible to record the whole emission spectra of both the sample and the reference.

\section{Octanol-water partitioning}

The octanol-water partition constant $\left(\mathrm{K}_{\mathrm{ow}}\right)$ was determined by dissolving LD-BTD1 in a 1:1 mixture of octanol and water by shaking vigorously and then left to separate to two phases. After separation, $2 \mathrm{ml}$ samples were taken from both phases. To obtain equivalent solvent conditions, $2 \mathrm{ml}$ water and $5 \mathrm{ml}$ methanol was added to the octanol sample and $2 \mathrm{ml}$ octanol and $5 \mathrm{ml}$ methanol was added to the water sample. Thus, the final solvent mixture of water:octanol:methanol was 2:2:5 for both samples. The octanol-water partition constant was then determined from the absorption spectra. The solubility of LD-BTD1 in water is low and to be able to compare the absorbance value of the two phases, the absorbance spectra for the water phase was measured in a 
$1 \mathrm{~cm}$ cuvette and the octanol phase in a $1 \mathrm{~mm}$ cuvette. Notably, the measured absorbance for the water phase was close to the sensitivity limit of the instrument, and the reported octanol/water partition coefficient is therefore best viewed as a lower estimate.

\section{pH dependence}

A small amount of LD-BTD1 was dissolved in DMSO and the resulting solution was diluted to a final DMSO concentration of 5\% using buffers with different $\mathrm{pH}(\mathrm{pH} 2$ and 4: Glycine $\mathrm{HCl}$ buffer, $50 \mathrm{mM}$; $\mathrm{pH} 6$ and 8: phosphate buffer, $25 \mathrm{mM}$; and $\mathrm{pH} 10$ and 12: Glycine $\mathrm{OH}$ buffer, $50 \mathrm{mM}$ ). Emission spectra were collected using a Tecan Saphire 2 micro plate reader (Tecan Group Ltd., Männerdorf, Switzerland). All measurements were repeated in triplicates and the excitation wavelength used was $450 \mathrm{~nm}$.

\section{Acknowledgements}

This work was supported by an international postdoctoral grant from the Swedish Research Council, Dnr: 350-2012-239 (to C.D), the Swedish Foundation of Strategic Research, ICA14-0018 (to K.B) and the Wenner-Gren Foundation (to K.S). The authors thank Professor Peter Strålfors at the Department of Clinical and Experimental Medicine, Linköping University for providing the adipocytes.

\section{Supporting information}

Supplementary data including NMR spectra, additional fluorescent microscopy pictures and photophysical characterization spectra can be found in the online version at doi...

\section{References and Notes}

(1) Thiam, A. R., Farese, R. V., Jr. and Walther, T. C. (2013) The biophysics and cell biology of lipid droplets. Nat. Rev. Mol. Cell Biol 14, 775.

(2) Guo, Y., Cordes, K. R., Farese, R. V. and Walther, T. C. (2009) Lipid droplets at a glance. J. Cell Sci. 122, 749.

(3) Martin, S. and Parton, R. G. (2006) Lipid droplets: a unified view of a dynamic organelle. Nat. Rev. Mol. Cell Biol. 7, 373.

(4) Thiele, C. and Spandl, J. (2008) Cell biology of lipid droplets. Curr. Opin. Cell Biol. 20, 378.

(5) Fujimoto, T. and Parton, R. G. (2011) Not just fat: the structure and function of the lipid droplet. Cold Spring Harb. Perspect Biol. 3.

(6) Beller, M., Thiel, K., Thul, P. J. and Jäckle, H. (2010) Lipid droplets: A dynamic organelle moves into focus. FEBS Lett. 584, 2176.

(7) Zehmer, J. K., Huang, Y., Peng, G., Pu, J., Anderson, R. G. and Liu, P. (2009) A role for lipid droplets in inter-membrane lipid traffic. Proteomics 9, 914.

(8) Boschi, F., Rizzatti, V., Zamboni, M. and Sbarbati, A. (2014) Lipid droplets fusion in adipocyte differentiated 3T3-L1 cells: a Monte Carlo simulation. Exp. Cell Res. 321, 201.

(9) Sun, Z., Gong, J., Wu, L. and Li, P. (2013) Imaging lipid droplet fusion and growth. Methods Cell Biol. 116, 253.

(10) Woscholski, R. (2006) Phospholipid signalling: mediators in need of interdisciplinary techniques. Signal Transduction 6, 77. 
(11) Krahmer, N., Farese, R. V. and Walther, T. C. (2013) Balancing the fat: lipid droplets and human disease. EMBO Mol. Med. 5, 973.

(12) Bozza, P. T. and Viola, J. P. (2010) Lipid droplets in inflammation and cancer. Prostaglandins Leukot. Essent. fatty acids 82, 243.

(13) Welte, M. A. (2015) Expanding roles for lipid droplets. Curr. Biol. 25, R470.

(14) Spiegelman, B. M. and Flier, J. S. Obesity and the Regulation of Energy Balance. Cell 104, 531.

(15) Fowler, S. D. and Greenspan, P. (1985) Application of Nile red, a fluorescent hydrophobic probe, for the detection of neutral lipid deposits in tissue sections: comparison with oil red O. J. Histochem. Cytochem. 33, 833.

(16) Greenspan, P. and Fowler, S. D. (1985) Spectrofluorometric studies of the lipid probe, nile red. J. Lipid Res. 26, 781.

(17) Greenspan, P., Mayer, E. P. and Fowler, S. D. (1985) Nile red: a selective fluorescent stain for intracellular lipid droplets. J. Cell Biol. 100, 965.

(18) Harris, L. A., Skinner, J. R. and Wolins, N. E. (2013) Imaging of neutral lipids and neutral lipid associated proteins. Methods Cell Biol. 116, 213.

(19) Spandl, J., White, D. J., Peychl, J. and Thiele, C. (2009) Live cell multicolor imaging of lipid droplets with a new dye, LD540. Traffic 10, 1579.

(20) Rumin, J., Bonnefond, H., Saint-Jean, B., Rouxel, C., Sciandra, A., Bernard, O., Cadoret, J. P. and Bougaran, G. (2015) The use of fluorescent Nile red and BODIPY for lipid measurement in microalgae. Biotechnol. Biofuels 8, 42.

(21) Gocze, P. M. and Freeman, D. A. (1994) Factors Underlying the Variability of Lipid Droplet Fluorescence in Ma-10 Leydig Tumor-Cells. Cytometry Part A 17, 151.

(22) Loudet, A. and Burgess, K. (2007) BODIPY dyes and their derivatives: syntheses and spectroscopic properties. Chem. Rev. 107, 4891.

(23) Wang, Z., Gui, C., Zhao, E., Wang, J., Li, X., Qin, A., Zhao, Z., Yu, Z. and Tang, B. Z. (2016) Specific Fluorescence Probes for Lipid Droplets Based on Simple AIEgens. ACS Appl. Mater. Interfaces 8, 10193.

(24) Sharma, A., Umar, S., Kar, P., Singh, K., Sachdev, M. and Goel, A. (2016) A new type of biocompatible fluorescent probe AFN for fixed and live cell imaging of intracellular lipid droplets. Analyst 141, 137.

(25) Goel, A., Sharma, A., Kathuria, M., Bhattacharjee, A., Verma, A., Mishra, P. R., Nazir, A. and Mitra, K. (2014) New fluoranthene FLUN-550 as a fluorescent probe for selective staining and quantification of intracellular lipid droplets. Org. Lett. 16, 756.

(26) Kim, E., Lee, S. and Park, S. B. (2012) A Seoul-Fluor-based bioprobe for lipid droplets and its application in image-based high throughput screening. Chem. Commun. 48, 2331.

(27) Gao, M., Su, H., Li, S., Lin, Y., Ling, X., Qin, A. and Tang, B. Z. (2017) An easily accessible aggregation-induced emission probe for lipid droplet-specific imaging and movement tracking. Chem. Commun. 53, 921.

(28) Jiang, D., Chen, S., Xue, Z., Li, Y., Liu, H., Yang, W. and Li, Y. (2016) Donoracceptor molecules based on benzothiadiazole: Synthesis, X-ray crystal structures, linear and third-order nonlinear optical properties. Dyes Pigm. 125, 100.

(29) Neto, B. A., Carvalho, P. H. and Correa, J. R. (2015) Benzothiadiazole Derivatives as Fluorescence Imaging Probes: Beyond Classical Scaffolds. Acc. Chem. Res. 48, 1560.

(30) Neto, B. A. D., Lapis, A. A. M., da Silva Júnior, E. N. and Dupont, J. (2013) 2,1,3-Benzothiadiazole and Derivatives: Synthesis, Properties, Reactions, and Applications in Light Technology of Small Molecules. Eur. J. Org. Chem. 2013, 228. 
(31) Carvalho, P. H., Correa, J. R., Guido, B. C., Gatto, C. C., De Oliveira, H. C., Soares, T. A. and Neto, B. A. (2014) Designed benzothiadiazole fluorophores for selective mitochondrial imaging and dynamics. Chem. Eur. J. 20, 15360.

(32) Tian, Y., Wu, W. C., Chen, C. Y., Jang, S. H., Zhang, M., Strovas, T., Anderson, J., Cookson, B., Li, Y., Meldrum, D., Chen, W. C. and Jen, A. K. (2010) Utilization of micelles formed from poly(ethylene glycol)-block-poly(epsilon-caprolactone) block copolymers as nanocarriers to enable hydrophobic red two-photon absorbing emitters for cells imaging. Journal of biomedical materials research. Part A 93, 1068. (33) da Cruz, E. H. G., Carvalho, P. H. P. R., Correa, J. R., Silva, D. A. C., Diogo, E. B. T., de Souza Filho, J. D., Cavalcanti, B. C., Pessoa, C., de Oliveira, H. C. B., Guido, B. C., da Silva Filho, D. A., Neto, B. A. D. and da Silva Junior, E. N. (2014) Design, synthesis and application of fluorescent 2,1,3-benzothiadiazole-triazolelinked biologically active lapachone derivatives. New. J. Chem. 38, 2569.

(34) Oliveira, F. F. D., Santos, D. C. B. D., Lapis, A. A. M., Corrêa, J. R., Gomes, A. F., Gozzo, F. C., Moreira Jr, P. F., de Oliveira, V. C., Quina, F. H. and Neto, B. A. D. (2010) On the use of 2,1,3-benzothiadiazole derivatives as selective live cell fluorescence imaging probes. Bioorg. Med. Chem. Lett. 20, 6001.

(35) Mota, A. A. R., Carvalho, P. H. P. R., Guido, B. C., de Oliveira, H. C. B., Soares, T. A., Correa, J. R. and Neto, B. A. D. (2014) Bioimaging, cellular uptake and dynamics in living cells of a lipophilic fluorescent benzothiadiazole at low temperature (4 [degree]C). Chemical Science 5, 3995.

(36) Bures, F. (2014) Fundamental aspects of property tuning in push-pull molecules. RSC Adv. 4, 58826.

(37) Mosmann, T. (1983) Rapid colorimetric assay for cellular growth and survival: application to proliferation and cytotoxicity assays. Journal of immunological methods 65, 55.

(38) Listenberger, L. L. and Brown, D. A., (2007) Fluorescent detection of lipid droplets and associated proteins. Current protocols in cell biology. Wiley \& Sons, Inc.: Vol. Chapter 24, p 24.2.1.

(39) Walther, T. C. and Farese, R. V., Jr. (2012) Lipid droplets and cellular lipid metabolism. Annu. Rev. Biochem. 81, 687.

(40) Koizume, S. and Miyagi, Y. (2016) Lipid Droplets: A Key Cellular Organelle Associated with Cancer Cell Survival under Normoxia and Hypoxia. Int. J. Mol. Sci. 17.

(41) Kuhajda, F. P. (2006) Fatty acid synthase and cancer: new application of an old pathway. Cancer Res. 66, 5977.

(42) Berry, R., Jeffery, E. and Rodeheffer, M. S. (2014) Weighing in on adipocyte precursors. Cell Metab 19, 8.

(43) Sudhoff, H., Schürmann, M. and Brotzmann, V. (2016) Autologous lipoinjection of the patulous Eustachian tube: Harvesting, cellular analysis, clinical application and preliminary outcome. Otorhinolaryngol Head Neck Sur. 2, DOI: $10.15761 /$ OHNS. 1000131.

(44) Rohwedder, A., Zhang, Q., Rudge, S. A. and Wakelam, M. J. (2014) Lipid droplet formation in response to oleic acid in Huh-7 cells is mediated by the fatty acid receptor FFAR4. J. Cell Sci. 127, 3104.

(45) Beloribi-Djefaflia, S., Vasseur, S. and Guillaumond, F. (2016) Lipid metabolic reprogramming in cancer cells. Oncogenesis 5, e189.

(46) Lakowicz, J. R., (2007) Principles of Fluorescence Spectroscopy. Springer US.

(47) Uchiyama, S., Kimura, K., Gota, C., Okabe, K., Kawamoto, K., Inada, N., Yoshihara, T. and Tobita, S. (2012) Environment-sensitive fluorophores with 
benzothiadiazole and benzoselenadiazole structures as candidate components of a fluorescent polymeric thermometer. Chem. Eur. J. 18, 9552.

(48) Gota, C., Uchiyama, S., Yoshihara, T., Tobita, S. and Ohwada, T. (2008) Temperature-Dependent Fluorescence Lifetime of a Fluorescent Polymeric Thermometer, Poly(N-isopropylacrylamide), Labeled by Polarity and Hydrogen Bonding Sensitive 4-Sulfamoyl-7-aminobenzofurazan. J. Phys. Chem. B 112, 2829.

(49) Ishi-i, T., Kitahara, I., Yamada, S., Sanada, Y., Sakurai, K., Tanaka, A., Hasebe, N., Yoshihara, T. and Tobita, S. (2015) Amphiphilic benzothiadiazoletriphenylamine-based aggregates that emit red light in water. Org. Biomol. Chem. 13, 1818.

(50) Sharma, A. K., Pavlova, S. T., Kim, J., Finkelstein, D., Hawco, N. J., Rath, N. P., Kim, J. and Mirica, L. M. (2012) Bifunctional compounds for controlling metalmediated aggregation of the abeta42 peptide. J. Am. Chem. Soc. 134, 6625.

(51) Dyrager, C., Vieira, R. P., Nystrom, S., Nilsson, K. P. R. and Storr, T. (2017) Synthesis and evaluation of benzothiazole-triazole and benzothiadiazole-triazole scaffolds as potential molecular probes for amyloid-[small beta] aggregation. New. J. Chem. 41, 1566.

(52) Danielsson, A., Öst, A., Lystedt, E., Kjolhede, P., Gustavsson, J., Nystrom, F. H. and Strålfors, P. (2005) Insulin resistance in human adipocytes occurs downstream of IRS1 after surgical cell isolation but at the level of phosphorylation of IRS 1 in type 2 diabetes. FEBS J. 272, 141.

(53) Sjöback, R., Nygren, J. and Kubista, M. (1995) Absorption and fluorescence properties of fluorescein. Spectrochim. Acta Mol. Biomol. Spectrosc. 51, L7. 